\title{
VALIDITY AND RELIABILITY OF THE KATZ-15 SCALE TO MEASURE UNFAVORABLE HEALTH OUTCOMES IN COMMUNITY-DWELLING OLDER PEOPLE
}

\author{
W. LAAN ${ }^{1}$, N.P.A. ZUITHOFF ${ }^{1}$, I. DRUBBEL ${ }^{1}$, N. BLEIJENBERG ${ }^{1}$, M.E. NUMANS ${ }^{1,2}$, \\ N.J. DE WIT ${ }^{1}$, M.J. SCHUURMANS ${ }^{3}$
}

\begin{abstract}
1. Department of General Practice, Julius Centre for Health Sciences and Primary Care, University Medical Centre Utrecht, Utrecht, The Netherlands; 2. Department of Public Health and Primary Care, Leiden University Medical Centre, Leiden, The Netherlands; 3. Department of Rehabilitation, Nursing Science and Sports Medicine, University Medical Centre Utrecht, Utrecht, The Netherlands. Corresponding author: Wijnand Laan, Assistant Professor, Julius Center for Health Sciences and Primary Care, University Medical Center Utrecht, Str. 6.131, P.O. Box 85500, 3508 GA Utrecht, The Netherlands
\end{abstract}

\begin{abstract}
Objectives: To predict the risk of future unfavourable health outcomes in older people it is common to assess the level of both basic and instrumental activities of daily living. To accomplish this, the commonly used Katz-6 and the Lawton IADL questionnaires can be combined to form the 'Modified Katz ADL' scale, also known as the Katz-15 scale. So far, the validity and reliability of the Katz-15 scale is unknown. The objective of the current study is to investigate how well the Katz-15 is able to predict future unfavorable health outcomes and how this is related to the existing Katz-6 scale. Design: We performed a follow-up study using data from a group of 60 year and older participants from a large Dutch clinical trial. Participants: We included 2321 participants in the analysis. The average age of the study population was 74 years and $44 \%$ was male. Measurements: We studied the relation between the Katz-15 scale and a number of unfavourable health outcomes, such as hospitalization, admission to a nursing home, admission to a home for the aged and death within one year of follow-up. Results: We found the Katz-15 to be both internally consistent and strongly associated with quality of life measures. We observed moderate to strong associations between the Katz-15 and the unfavourable health outcomes All associations studied were stronger for the Katz-15 scale as compared to the Katz-6 scale. Conclusion: The results of our study indicate that the Katz-15 scale is able to reliably and validly predict future unfavorable health outcomes. This makes the scale a valuable measure in determining both basic and instrumental activities of daily living
\end{abstract}

Key words: Independence, Katz-15, Katz-6.

\section{Introduction}

The number of older people in society is increasing worldwide (1). This increase is especially notable in the Western Europe where the large group of post-World War II born 'Baby-boomers', is now reaching the age of 65 . With increasing age people are more likely to develop chronic disorders and lose independence. Increasing use of healthcare, medication and (in the end) referral to institutionalized care are the consequence of decreasing independency. For this reason an important aim in the care for older people is to maintain the levels of independence and postpone any decline. To measure the level of independence several measures can be used. The best known in clinical practice and clinical research are the Katz-6 ADL index (Katz-6) (2) and the Lawton Instrumental Activities of Daily Living index (LIADL) (3). Both the Katz-6 and the LIADL assess important aspects of the levels of independence in older people, but in different domains. The Katz- 6 assesses basic activities of daily living such as the need for help to go to the toilet or for assistance in eating but it does not assess instrumental activities of daily living and ADLs alone are found to underestimate dysfunction and disability (4). The LIADL assesses everyday functional, or instrumental, competence such as whether one needs help in shopping or taking care of the household, but it does not include the basic activities of daily living that the Katz- 6 does. Also, the Katz-6 and the LIADL are not considered to be unidimensional and in a study by Thomas et al. it is advised that the these scales should be considered in combination to capture a greater range of functional disability prevalence (4). To assess both basic and instrumental aspects independence the Katz- 6 and the LIADL are therefore often combined, which is then referred to as the 'Modified Katz ADL', or Katz-15 scale. The Katz-15 is frequently used in clinical research (5-9), especially recently in the Dutch 'National Care for the Elderly Programme' (10). In this programme a total of 62 projects participate with the aim to improve care and support for older people with complex care needs. Many of these projects use the Katz-15 scale as an important outcome in assessing the effectiveness of the interventions. The katz-15 is sometimes also referred to as the 'adjusted Katz'. Until now however, the Katz-15 scale has only very briefly been described (11) and the reliability and validity of this scale has never been investigated. We want to emphasise that this study does not add another measure of ADL to the literature but rather to further investigate and describe existing scale. The primary aim of this study is therefore to investigate the reliability (internal consistency) and validity of the Katz15 scale in regards to other well-known health related scales. Additionally, we assessed the predictive ability of the Katz-15 for unfavourable health outcomes in a group of community 
dwelling older people with multiple chronic disorders. Predictive validity is important as the Katz- 15 should be able to predict a subsequent deterioration in health status to be a clinically relevant measure. For comparison, we also studied the validity of the Katz- 6 . The secondary aim of this paper is to present the items of the Katz-15 scale and demonstrate the distribution of its scores in a population to which it is targeted.

\section{Methods}

\section{Study population}

This study used data collected in the Dutch U-PROFIT trial, part of the 'National Care for the Elderly Programme'. The UPROFIT trial is a single-blind, three-armed, cluster-randomized controlled trial (RCT) on proactive elderly care in community dwelling frail elderly with one year follow-up. This RCT has been described in detail elsewhere (12). In short the U-PROFIT trial was designed to evaluate the effectiveness of a screening and monitoring intervention using routine healthcare data and a nurse-led multidisciplinary intervention program to care as usual (12). The study population included frail participants of 60 years and over who were recruited at general practitioner (GP) centres from the Utrecht region in the Netherlands. To exclude those from the U-PROFIT trial with a relatively low risk for functional decline we included only those participants in the current study who used five or more types of medication. Polypharmacy is taken as a proxy for multimorbidity. This excluded $18 \%$ of the U-PROFIT participants who used less than five different types of medication.

\section{The Katz-15 questionnaire}

The first six items of the Katz-15 questionnaire are equal to the items of the Katz- 6 scale and assess the ability of an individual to independently bath, dress, use a toilet, transfer to and from a chair, the use of incontinence products and the ability to eat without help (table 1). Questions seven to thirteen are seven out of a total of eight questions from the Lawton IADL scale. These items address whether a person needs help to use a telephone, to go shopping, to prepare food, to perform household tasks, to travel, to take medication and to handle own finances. The Lawton IADL item that assesses whether one is able to do his own laundry was excluded from this list as most people in western European countries no longer do the laundry by hand, but have a washing machine or take the laundry to a laundrette. The two last items in the Katz-15 ask whether one needs help with brushing and combing hair or shaving and whether one needs help walking about. These items were not derived from either the Katz- 6 or the Lawton IADL. For the complete list of questions in the Katz-15, see table 1 .

\section{Outcome}

For the Katz 15 to be a valid index for activities of daily living, we choose four unfavourable health outcomes after one year of follow-up which the Katz-15 had to be able to adequately predict. These outcomes were hospitalisation, admission to a home for the aged, admission to a nursing home and death. Hospitalisation, admission to a home for the aged and admission to a nursing home were assessed using questionnaires. All answers to these questions were double checked for validity in the electronical medical records (EMR) of the treating GP of the participants. Information on whether a participant died during the twelve months of follow-up was extracted both from the EMR and correspondence with relatives of the participants. Other characteristics such as demographics, co-comorbidity, the SF-36 items and the EQ-5C and EQ5D

Table 1

The Katz-15 items and their source

\begin{tabular}{|c|c|c|c|c|}
\hline Katz-15 item & Question & Source & Yes & No \\
\hline 1 & Do you need help with taking a bath or a shower? & Katz-6 & 1 & 0 \\
\hline 2 & Do you need help getting dressed? & Katz-6 & 1 & 0 \\
\hline 3 & Do you need help toileting? & Katz-6 & 1 & 0 \\
\hline 4 & Do you need help sitting down and getting up from a chair? & Katz-6 & 1 & 0 \\
\hline 5 & Do you use incontinence products? & Katz-6 & 1 & 0 \\
\hline 6 & Do you need help with eating? & Katz-6 & 1 & 0 \\
\hline 7 & Do you need help using the telephone? & Lawton & 1 & 0 \\
\hline 8 & Do you need help shopping? & Lawton & 1 & 0 \\
\hline 9 & Do you need help preparing a meal? & Lawton & 1 & 0 \\
\hline 10 & Do you need help taking care of your house? & Lawton & 1 & 0 \\
\hline 11 & Do you need help travelling? & Lawton & 1 & 0 \\
\hline 12 & Do you need help taking your medications? & Lawton & 1 & 0 \\
\hline 13 & Do you need help handling your finances? & Lawton & 1 & 0 \\
\hline 14 & Do you need help brushing your hair or shaving? & Extra & 1 & 0 \\
\hline 15 & Do you need help walking about? & Extra & 1 & 0 \\
\hline
\end{tabular}


were extracted from the U-PROFIT questionnaires returned at baseline. The number of chronically prescribed medications and the number of GP visits were extracted from the EMRs of the treating GPs.

\section{Analysis}

The reliability (internal consistency) of the Katz-15 scores was assessed with Kuder-Richardson-20 measure (KR-20)(13). The KR-20 is similar to Cronbach's alfa, however assessing the internal consistency of dichotomous items. Further assessment of the validity was performed in two steps. We first estimated Spearman rank correlations between the Katz-15 and other health related well-being indices such as the physical, mental, social and vitality subscales of the SF-36(14) and the EQ5-D and EQ5-C scales (15), as well as more objective health problem related measures, such as the Frailty index (16). All these scales were measured at inclusion. Second, we constructed ROC curves and estimated corresponding areasunder-the-curves (AUC) to assess the ability of Katz-15 to predict the primary outcomes, being hospitalisation, admission to a home for the aged, admission to a nursing home and death within the twelve months of follow-up. Neither of the analyses performed in this study included other covariates such as age, gender and comorbidities. As a reference we will also present the AUCs of the model including only the count of comorbid disorders on the four outcomes of interest.

\section{Results}

\section{Participants and covariates}

A total of 3092 people were included in the U-PROFIT trial (Table 2). Of those, $2573(82 \%)$ participants were polypharmacy users. After excluding those with missing data on gender (101 participants) or Katz-6 or Katz-15 questionnaires (151 participants), data from 2321 participants could be included in the analysis. The average age of the study population was 74 years and $44 \%$ was male. The distribution of both the Katz-15 and the Katz- 6 was highly skewed towards the lower end of scores (table 3). Median scores on the Katz-15 and Katz- 6 were 1 and 0 , with inter quartile ranges (IQR) of 0 3 and $0-1$ ) respectively. On the Katz-15, $43 \%$ of patients reported no need for help on any of the items, whereas $67 \%$ of patients answered none of the Katz-6 items positively.

The number of patients per Katz-15 category and Katz-6 category declined with each incline of Katz-15 and Katz-6 severity score. The internal consistency of the Katz-15 and Katz- 6 as estimated with the KR-20 were 0.80 and 0.55 respectively. The number and percentages of men and women that answer positive to Katz- 15 and Katz- 6 items are presented in table 4.

\section{Correlation of the Katz-15 with other health indicators}

The correlation between the Katz-15 and the SF-36 scales varied from -0.24 to -0.72 (Table 5). Correlations with the frailty index and the number of prescribed medications was 0.27 and 0.29 respectively. The correlation with the EQ-5D was found to be strong with -0.59 . For comparison, all associations were consistently lower for the KATZ-6 scale (Table 5).

\section{Table 2}

Patient characteristics and outcome, total / percentages or mean \pm standard deviation

\begin{tabular}{lc}
\hline Characteristic & $\mathbf{N}(\boldsymbol{\%})$ \\
\hline Total patients & 2321 \\
Male gender & $1010 / 44 \%$ \\
Age & $74 / 8$ \\
Followed education after 14 years of age & $1575 / 68 \%$ \\
Frailty Index & $0.08 \pm 0.05$ \\
Comorbidity & \\
Diabetes & $764 / 33 \%$ \\
Depression & $178 / 8 \%$ \\
Stroke, TIA & $207 / 9 \%$ \\
Heart Failure & $575 / 25 \%$ \\
Malignant disease & $222 / 10 \%$ \\
Asthma/COPD & $568 / 25 \%$ \\
Dementia & $24 / 1 \%$ \\
Number of prescribed medication & $7 \pm 2$ \\
SF-36 Physical & $57 \pm 29$ \\
SF-36 Mental & $69 \pm 19$ \\
SF-36 Social & $43 \pm 11$ \\
SF-36 Vitality & $56 \pm 20$ \\
EQ-5D & $0.74 \pm 0.23$ \\
EQ-5C & $1.34 \pm 0.50$ \\
Hospitalisation & $696 / 35 \%$ \\
Admission to a nursing home & $31 / 2 \%$ \\
Admission to a home for the aged & $42 / 2 \%$ \\
Death & $66 / 3 \%$ \\
\hline
\end{tabular}

\section{Relation to patient health outcomes}

During the twelve months of follow-up 696 (36\%) participants were hospitalized, $31(2 \%)$ participants were admitted to a nursing home, $42(2 \%)$ participants were admitted to a home for the aged and $66(3 \%)$ participants died. The ROC-curves for the association between the Katz-15 and hospitalization, admission to a nursing home, admission to a home for the aged and death can be seen in figure 1. We found AUCs for these associations of 0.61 (95\% Confidence Interval (95\%-CI): 0.59 -0.64), 0.86 (95\%-CI: $0.82-0.91), 0.83$ (95\%CI: $0.77-0.89)$ and 0.69 (95\%-CI: $0.62-0.77)$, respectively. For the Katz-6 these AUCs were 0.58 (95\%-CI: 0.56 - 0.60), 0.78 (95\%-CI: $0.69-0.86), 0.76(95 \%-C I: 0.69-0.84)$ and 0.67 (95\%-CI: $0.60-0.74)$. The AUCs for the model with only the total count of comorbid disorders were 0.58 (95\%-CI: 0.56 - 0.61$), 0.65$ (95\%-CI: $0.54-0.77), 0.69$ (95\%-CI: $0.60-$ $0.77)$ and 0.62 (95\%-CI: $0.55-0.89)$, respectively. 
Table 3

Number and percentages of men and women for all Katz-15 and Katz-6 total scores

\begin{tabular}{lcccc}
\hline Total score & $\begin{array}{c}\text { Katz-15, men } \\
(\mathbf{N} / \%)\end{array}$ & $\begin{array}{c}\text { Katz-15, women } \\
(\mathbf{N} / \%)\end{array}$ & $\begin{array}{c}\text { Katz-6, men } \\
(\mathbf{N} / \%)\end{array}$ & $\begin{array}{c}\text { Katz-6, women } \\
(\mathbf{N} / \%)\end{array}$ \\
\hline 0 & $625 / 62 \%$ & $369 / 28 \%$ & $818 / 81 \%$ & $739 / 56 \%$ \\
1 & $18518 \%$ & $281 / 21 \%$ & $120 / 12 \%$ & $382 / 29 \%$ \\
2 & $62 / 6 \%$ & $188 / 14 \%$ & $31 / 3 \%$ & $107 / 8 \%$ \\
3 & $41 / 4 \%$ & $143 / 11 \%$ & $23 / 2 \%$ & $49 / 4 \%$ \\
4 & $27 / 3 \%$ & $125 / 10 \%$ & $9 / 1 \%$ & $8 / 2 \%$ \\
5 & $23 / 2 \%$ & $79 / 6 \%$ & $4 / 0 \%$ & $4 / 0 \%$ \\
6 & $17 / 2 \%$ & $59 / 5 \%$ & $5 / 1 \%$ & - \\
7 & $9 / 1 \%$ & $34 / 3 \%$ & - & - \\
8 & $7 / 1 \%$ & $13 / 1 \%$ & - & - \\
9 & $2 / 0 \%$ & $7 / 1 \%$ & - & - \\
10 & $3 / 0 \%$ & $5 / 0 \%$ & - & - \\
11 & $6 / 1 \%$ & $2 / 0 \%$ & - & - \\
12 & $3 / 0 \%$ & $3 / 0 \%$ & - & - \\
13 & $2 / 0 \%$ & - & - \\
14 & $0 / 0 \%$ & $1 / 0 \%$ & - & - \\
15 & $0 / 0 \%$ & $0 / 0 \%$ & & - \\
\hline
\end{tabular}

Table 4

Number and percentages of men and women that answer positive to Katz-15 and Katz- 6 items. Item numbers correspond to the item numbers in table 1

\begin{tabular}{|c|c|c|c|}
\hline Katz-15 item & Katz-6 item & $\begin{array}{c}\text { Number of men with positive } \\
\text { answer }(\mathrm{N} / \%)\end{array}$ & $\begin{array}{c}\text { Number of women with positive } \\
\text { answer }(\mathrm{N} / \%)\end{array}$ \\
\hline 1 & 1 & $62 / 6 \%$ & $143 / 11 \%$ \\
\hline 2 & 2 & $51 / 51 \%$ & 97 / 7\% \\
\hline 3 & 3 & $11 / 1 \%$ & $16 / 1 \%$ \\
\hline 4 & 4 & $34 / 3 \%$ & 75 / $6 \%$ \\
\hline 5 & 5 & $76 / 8 \%$ & $440 / 33 \%$ \\
\hline 6 & 6 & $3 / 0 \%$ & $7 / 1 \%$ \\
\hline 7 & - & $33 / 3 \%$ & $30 / 2 \%$ \\
\hline 8 & - & $114 / 11 \%$ & $447 / 34 \%$ \\
\hline 9 & - & 99 / 10\% & $128 / 10 \%$ \\
\hline 10 & - & $271 / 27 \%$ & $811 / 61 \%$ \\
\hline 11 & - & $129 / 13 \%$ & $439 / 34 \%$ \\
\hline 12 & - & $38 / 4 \%$ & $49 / 4 \%$ \\
\hline 13 & - & $3 / 0 \%$ & $5 / 0 \%$ \\
\hline 14 & - & $10 / 1 \%$ & $21 / 2 \%$ \\
\hline 15 & - & $65 / 7 \%$ & $207 / 16 \%$ \\
\hline
\end{tabular}

Table 5

Spearman rank correlations between Katz-15 scores, Katz-6 scores and health measurements

\begin{tabular}{lcc}
\hline Health measurement & Katz-15 & Katz-6 \\
\hline SF-36 Physical & -0.72 & -0.52 \\
SF-36 Mental & -0.35 & -0.27 \\
SF-36 Social & -0.24 & -0.17 \\
SF-36 Vitality & -0.48 & -0.37 \\
EQ-5D & -0.59 & -0.46 \\
Frailty Index & 0.27 & 0.21 \\
Number of prescribed medication & 0.29 & 0.22 \\
\hline
\end{tabular}

Note: The correlations between the Katz-scales and quality of life (SF-36 scales and EQ5D) are negative due to reversed scales: for example higher Katz scores indicate a lowe ADL, whereas higher SF-36 scores indicate a better quality of life.

\section{Discussion}

\section{Summary of findings}

Using data from a large clinical trial in elderly participants we found the Katz-15 to be both internally consistent with a KR-20 of 0.80 and weak to strong associated with other quality of life measures with correlations ranging from -0.24 to -0.72 .

Although the number of participants with Katz-15 scores of 7 or higher was low, the main advantage over the Katz-6 appeared to be the smaller percentage of participants with a score of zero as compared to the Katz-6 (table 3). The association of the Katz-15 with the frailty index and the number of prescribed medications was weak, 0.27 and 0.21 respectively. We observed moderate to strong associations between the Katz-15 and unfavourable health outcomes such as 


\section{Figure 1}

ROC-curves for the association between the Katz-15/6 and hospitalisation, admission to a nursing home, admission to a home for the aged and death after twelve months
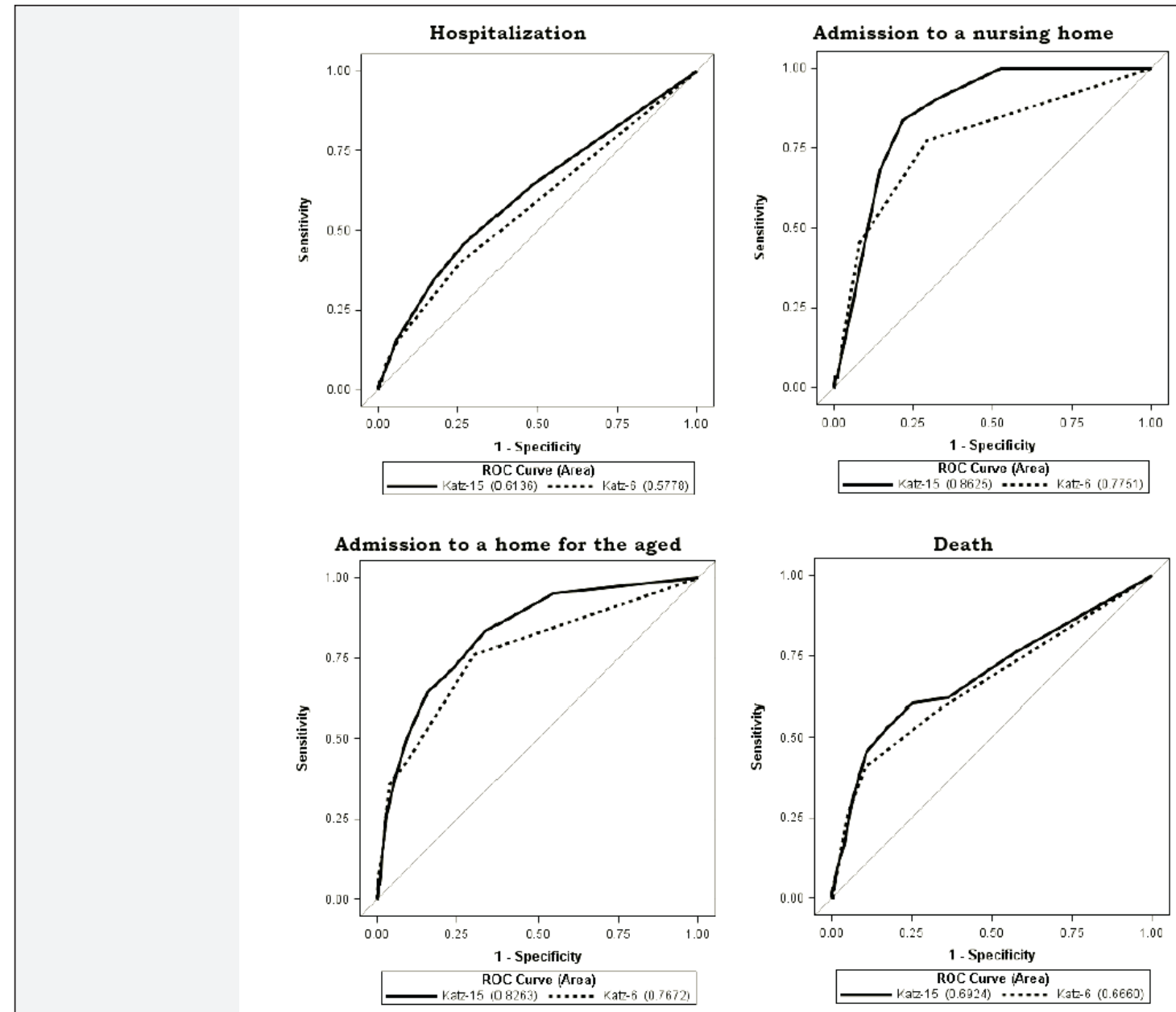

hospitalization, admission to a nursing home, admission to a home for the aged and death with AUCs in the range of 0.61 to 0.86. These high to moderate AUCs indicate that the Katz-15 has good predictive validity and appears to be a good indicator for deteriorating health in elderly patients.

The AUCs for the Katz-15 scale were all higher as compared to a model with only the count of comorbid disorders. This was most notable for the outcomes 'admission to a nursing home' and 'admission to a home for the aged'. This indicates that these negative health outcomes are more closely associated with activities of daily living than with determinants of physical and mental health.

The associations between the Katz-6 and all quality of life measures as well as health care consumption indicators was clearly lower when compared to the Katz-15. These findings underline the additional value of the extra items in the Katz- 15 . This was also observed as the Katz- 15 was better able to predict unfavourable health outcomes. The association of the Katz-15 with the frailty index was lower than expected.

\section{Explanation of findings}

The higher association between the Katz-15 and unfavourable healthcare outcomes as compared to the Katz-6 can be explained by the additional instrumental items that are included in the Katz-15 scale. For example, item 10 assesses whether one is able to take care his or her house without help. A total of $60 \%$ of women gave a positive answer to this question. As $58 \%$ of the women had a score of 0 on the Katz- 6 scale, whereas this was only $30 \%$ for the Katz- 15 scale it is likely that this item is an important discriminator in the Katz-15 scale.

We found a relatively high KR-20 for the Katz- 15 as relative to the Katz-6. The explanation for this finding is the substantial increase in the number of items in the Katz-15, more than twice the number of items in the Katz-6. This in turn resulted in a different, more scattered distribution of the sum score of the Katz-15 when compared to the Katz-6, or, in more technical terms, a higher variance.

Because the participants in the current study were relatively 
independent, the Katz-6 scale showed relatively poor discrimination on our outcomes of interest. By using the Katz15 scale, which adds additional items to the Katz-6, we were able to broaden the spectrum of possible scores and made the distributions of total scores less skewed towards the scores of zero and one. This resulted in better discrimination between those with and without our outcome of interest.

\section{Strengths and limitations}

To appreciate the findings from this paper a few issues need to be addressed.

First, we evaluated the validity of the Katz-15 in data from a clinical trial with a high number of participants. This allowed for comparison with established quality of life measurements as well as assessing the validity against highly relevant clinical outcomes in one year follow-up.

Also, the Katz-15 scale that we investigated in our study consists of only 15 items. The items are easily assessed and don't require a lot of time to answer.

As the study population did not include elderly living in homes of the aged the study population may be relatively healthy compared to the total Dutch population of elderly. On the other hand all the participants in the study had to chronically use five or more different types of medication, so the population might as well be relatively unhealthy. The results from our study regarding death during follow-up might therefore not be generalizable any population, such as the relatively healthy elderly. The first point is also reflected by the relatively low percentages of unfavourable outcomes in our study. Of all 2321 participants only $36 \%, 2 \%, 2 \%$ and $3 \%$ were respectively hospitalized, admitted to a nursing home, to a home for the aged or died during follow-up. Although the number of participants in the most independent groups was relatively high for the Katz-15 scale relative to the Katz- 6 scale, the absolute numbers in the more dependent groups remain relatively low. Only $1 \%$ of the study population had a Katz-15 score of 9 or higher. Therefore, the added value of the Katz-15 over the Katz-6 seems especially strong in in the most independent participants.

An important strength of the study is the fact that it also provides evidence of criterion validity for the Katz-15 by the comparison to other measures of interest like the SF-36 and the number of medications.

A minor weakness of the Katz-15 is the fact that was not very well able to predict hospitalisation, as we found an AUC of 0.61 (95\%-CI: $0.59-0.64)$. This is unfortunate since hospitalisation itself is also known to be a risk factor for subsequent further functional decline (17).

\section{Comparison with similar measures}

Besides the Katz-15, the Katz-6 and the LIALD there are several other scales which are used to assess the levels of independence in the elderly. Among these are the lesser known Barthel index(18) and the Functional Independence Measure
(FIM) (19).

The Barthel index (18) assess ten items. Eight out of the ten items from this scale are also included in the Katz-15. The two items which are not in the Katz-15 are whether one is able to control his or her bowels and whether one is able to ascend and descend stairs.

Another scale is the Functional Independence Measure (FIM) (19) which includes 18 items, of which 13 items address the physical domain, based on the Barthel Index, and 5 items address the cognitive domain. These five cognitive items assess problem-solving skills, memory, orientation, attention and safety judgement. The Katz-15 scale does not include cognitive aspects of independence but only the physical aspects. Comparison of the Katz-15 to the FIM may therefore be difficult.

Other, even lesser known scales used in assessing the levels independence in the elderly, are the Care Needs Assessment (CNA) (20) and the Donaldson ADL Evaluation Form(21), although it seems they are not actively used in clinical practice anymore, and finding information about these scales seems difficult.

When comparing the Katz-15 to the Katz-6, the Lawton IADL, the Barthel index and the FIM we feel that the Katz-15 is a very valuable addition to the already available measures currently used as it combines multiple domains assessed by the individual scales.

\section{Conclusion}

The results of our study indicate that the Katz-15 scale is a reliable and valid measurement of ADL. Extending the Katz-6 with the extra items from the LIADL to form the Katz-15 increases the variability, reliability and predictive validity. These points, combined with the simple, easy-to-use format, makes the Katz-15 an attractive instrument for use by healthcare professionals.

Sponsor: This work was supported by a grant from The Netherlands Organization for Health Research and Development (grant number 311040201).

\section{References}

1. United Nations: Department of Economic and Social Affairs. World Population Ageing 2007., 2007 Edition ed., United Nations Publications.

2. Katz, S., Ford, A. B., Moskowitz, R. W., Jackson, B. A. \& Jaffe, M. W. Studies of Illness in the Aged. JAMA: The Journal of the American Medical Association 1963;185: 914-919.

3. Lawton, M. P. \& Brody, E. M. Assessment of older people: self-maintaining and instrumental activities of daily living. Gerontologist. 19639;9: 179-186.

4. Thomas, V. S., Rockwood, K. \& McDowell, I. Multidimensionality in Instrumental and Basic Activities of Daily Living. Journal of Clinical Epidemiology 1998;51: 315321.

5. Suijker, J. J., Buurman, B. M., ter, R. G., van, R. M., de Haan, R. J., de Rooij, S. E. \& Moll van Charante, E. P. Comprehensive geriatric assessment, multifactorial interventions and nurse-led care coordination to prevent functional decline in community-dwelling older persons: protocol of a cluster randomized trial. BMC. Health Serv. Res. 2012;12:85. doi: 10.1186/1472-6963-12-85.: 85-12.

6. Makai, P., Koopmanschap, M. A., Brouwer, W. B. \& Nieboer, A. A. A validation of the ICECAP-O in a population of post-hospitalized older people in the Netherlands. Health Qual. Life Outcomes. 2013;11:57. doi: 10.1186/1477-7525-11-57.: 57-11. 


\section{VALIDITY AND RELIABILITY OF THE KATZ-15 SCALE TO MEASURE UNFAVORABLE HEALTH OUTCOMES}

7. Laan, W., Bleijenberg, N., Drubbel, I., Numans, M. E., de Wit, N. J. \& Schuurmans, M. J. Factors associated with increasing functional decline in multimorbid independently living older people. Maturitas. 2013;75: 276-281.

8. den Ouden, M. E. M., Schuurmans, M. J., Mueller-Schotte, S., Brand, J. S. \& van der Schouw, Y. T. Domains Contributing to Disability in Activities of Daily Living. Journal of the American Medical Directors Association 2013;14: 18-24.

9. Ruikes, F. G., Meys, A. R., van de, W. G., Akkermans, R. P., van Gaal, B. G., Zuidema, S. U., Schers, H. J., van, A. T. \& Koopmans, R. T. The CareWell-primary care program: design of a cluster controlled trial and process evaluation of a complex intervention targeting community-dwelling frail elderly. BMC. Fam. Pract. 2012;13:115. doi: 10.1186/1471-2296-13-115.: 115-13.

10. ZonMw. The National Care for the Elderly Programme - abridged programme document. 1-8. 2008.

11. Weinberger, M., Samsa, G. P., Schmader, K., Greenberg, S. M., Carr, D. B. \& Wildman, D. S. Comparing proxy and patients' perceptions of patients' functional status: results from an outpatient geriatric clinic. J. Am. Geriatr. Soc. 1992;40: 585588.

12. Bleijenberg, N., Drubbel, I., ten Dam, V., Numans, M., Schuurmans, M. \& de Wit, N. Proactive and integrated primary care for frail older people: Design and methodological challenges of the Utrecht Primary care PROactive Frailty Intervention Trial (U-PROFIT). BMC Geriatrics 2012;12: 16.

13. Crocker, L. M. \& Algina, J. Introduction to classical and modern test theory. Holt, Rinehart, and Winston, New York, 1986.
14. Ware, J. E., Jr. \& Sherbourne, C. D. The MOS 36-Item Short-Form Health Survey (SF-36): I. Conceptual Framework and Item Selection. Medical Care 1992;30: 473483.

15. Krabbe, P. F. M., Stouthard, M. E. A., Essink-Bot, M. L. \& Bonsel, G. J. The Effect of Adding a Cognitive Dimension to the EuroQol Multiattribute Health-Status Classification System. Journal of Clinical Epidemiology 1999;52: 293-301.

16. Drubbel, I., de Wit, N. J., Bleijenberg, N., Eijkemans, R. J. C., Schuurmans, M. J. \& Numans, M. E. Prediction of Adverse Health Outcomes in Older People Using a Frailty Index Based on Routine Primary Care Data. The Journals of Gerontology Series A: Biological Sciences and Medical Sciences 2013;68: 301-308.

17. Lakhan, P., Jones, M., Wilson, A. \& Gray, L. C. The decline in Activities of Daily Living at Discharge (DADLD) index: stratifying patients at lower and higher risk. J. Nutr. Health Aging. 2012;16: 919-924.

18. Mahoney, F. I. \& Barthel, D. W. Functional evaluation: The BARTHEL index. Md State Med. J. 1965;14:61-5.: 61-65.

19. Keith, R. A., Granger, C. V., Hamilton, B. B. \& Sherwin, F. S. The functional independence measure: a new tool for rehabilitation. Adv. Clin. Rehabil. 1987;1:618.: 6-18.

20. Hirsch, C. H., Sommers, L., Olsen, A., Mullen, L. \& Winograd, C. H. The natural history of functional morbidity in hospitalized older patients. J. Am. Geriatr. Soc. 1990;38: 1296-1303.

21. Donaldson, S. W., Wagner, C. C. \& Gresham, G. E. A unified ADL evaluation form. Arch. Phys. Med. Rehabil. 1973;54: 175-179. 